\title{
Identification and characterization of miRNAs and targets in flax (Linum usitatissimum) under saline, alkaline, and saline-alkaline stresses
}

Ying $\mathrm{Yu}^{1,2}$, Guangwen $\mathrm{Wu}^{2}$, Hongmei Yuan ${ }^{1,2}$, Lili Cheng ${ }^{2}$, Dongsheng Zhao ${ }^{2}$, Wengong Huang ${ }^{2}$, Shuquan Zhang ${ }^{2}$, Liguo Zhang ${ }^{2}$, Hongyu Chen ${ }^{3}$, Jian Zhang ${ }^{4^{*}}$ and Fengzhi Guan ${ }^{1,2^{*}}$

\begin{abstract}
Background: MicroRNAs (miRNAs) play a critical role in responses to biotic and abiotic stress and have been characterized in a large number of plant species. Although flax (Linum usitatissimum L.) is one of the most important fiber and oil crops worldwide, no reports have been published describing flax miRNAs (Lus-miRNAs) induced in response to saline, alkaline, and saline-alkaline stresses.

Results: In this work, combined small RNA and degradome deep sequencing was used to analyze flax libraries constructed after alkaline-salt stress (AS2), neutral salt stress (NSS), alkaline stress (AS), and the non-stressed control (CK). From the CK, AS, AS2, and NSS libraries, a total of 118, 119, 122, and 120 known Lus-miRNAs and 233, 213, 211, and 212 novel Lus-miRNAs were isolated, respectively. After assessment of differential expression profiles, 17 known Lus-miRNAs and 36 novel Lus-miRNAs were selected and used to predict putative target genes. Gene ontology term enrichment analysis revealed target genes that were involved in responses to stimuli, including signaling and catalytic activity. Eight Lus-miRNAs were selected for analysis using qRT-PCR to confirm the accuracy and reliability of the miRNA-seg results. The qRT-PCR results showed that changes in stress-induced expression profiles of these miRNAs mirrored expression trends observed using miRNA-seq. Degradome sequencing and transcriptome profiling showed that expression of 29 miRNA-target pairs displayed inverse expression patterns under saline, alkaline, and saline-alkaline stresses. From the target prediction analysis, the miR398a-targeted gene codes for a copper/zinc superoxide dismutase, and the miR530 has been shown to explicitly target WRKY family transcription factors, which suggesting that these two micRNAs and their targets may significant involve in the saline, alkaline, and saline-alkaline stress response in flax.

Conclusions: Identification and characterization of flax miRNAs, their target genes, functional annotations, and gene expression patterns are reported in this work. These findings will enhance our understanding of flax miRNA regulatory mechanisms under saline, alkaline, and saline-alkaline stresses and provide a foundation for future elucidation of the specific functions of these miRNAs.
\end{abstract}

Keywords: MicroRNAs, Saline-alkaline stress, Deep sequencing, Degradome, Flax

\footnotetext{
* Correspondence: jian.zhang@albertainnovates.ca; kj-gfz@163.com

${ }^{4}$ Alberta Innovates Technology Futures, Vegreville, Alberta T9C 1 T4, Canada

${ }^{1}$ Heilongjiang Academy of Agricultural Sciences Postdoctoral Programme,

Harbin 150086, People's Republic of China

Full list of author information is available at the end of the article
} 


\section{Background}

Salt stress is one of the major environmental stresses that limit worldwide agricultural crop yields and will continue to be of concern in future years. In responses to salt stress, such as ionic and osmotic stress, crops have evolved multiple molecular networks to regulate homeostasis and maintain their growth and development. Exposure to salt stress triggers cascades of signal transduction pathways, which induces changes in gene expression profiles [1]. Alkaline-salt stress is generally associated with $\mathrm{NaHCO}_{3}$ or $\mathrm{Na}_{2} \mathrm{CO}_{3}$ presence and crops growing in alkaline soils suffer from both high $\mathrm{pH}$ stress and $\mathrm{CO}_{3}^{2-} / \mathrm{HCO}_{3}^{-}$stress [2]. Therefore, understanding of the saline-alkaline stress response may help to improve crop tolerance to salt stress. However, the mechanisms of plant alkaline-salt tolerance are poorly understood.

MicroRNAs (miRNAs) are endogenous 19-24 nt stretches of noncoding single-stranded RNA that negatively regulate gene expression by inhibiting gene translation or by promoting cleavage of target mRNAs post-transcriptionally [3]. The miRNAs were first discovered in Caenorhabditise legans in 1993 [4] and the first plant miRNAs were identified in Arabidopsis in 2002 [5]. Recently, several researchers have shown that miRNAs play important roles in plant responses to various abiotic stresses, including low temperature [6], drought [7], high salinity [8], oxidative [9], hypoxic [10], UV-B radiation [11], and metals stress [12]. Additionally, several studies have shown that many differentially expressed miRNAs and their target mRNAs are involved in adaptation to salt stress environments [13]. MiR393 was strongly upregulated when Arabidopsis was treated with $300 \mathrm{mM}$ $\mathrm{NaCl}$, while miR398 was down-regulated under salt stress $[14,15]$. In rice, miR169g was shown to be upregulated during high-salinity stress [16]. Moreover, transgenic rice plants that over-expressed miR393 and miR396c were more sensitive to salt stress $[17,18]$. For a large number of plant species, it is becoming increasingly evident that miRNAs play an important role in plant salt stress. Therefore, more studies on miRNA expression in response to salt stress in plants are required. Furthermore, more research at the genome level using high-throughput sequencing methodologies should facilitate future discovery of additional alkaline-salt stress responsive miRNAs in plants.

Flax (Linum usitatissimum) is a member of the genus Linum in the family Linaceae and is grown as a food and fiber crop worldwide. Attempts have been made to grow flax in saline-alkaline soil in order to avoid competition for land with other food crops with limited success. Achieving better yields would greatly improve flax fiber supply and foster sustainable development practices in the flax industry. Therefore, using different strategies, flax breeders have made great efforts to develop a salt tolerant flax cultivar $[19,20]$. However, the successful cultivation of salt tolerant flax varieties has not yet been reported. Fortunately, the recent release of the flax genome sequence has furthered understanding of transcriptional level molecular mechanisms of flax adaptation to saline-alkaline stress [21]. Moreover, digital gene expression has recently resulted in identification of several differentially expressed genes in flax under saline-alkaline stress [22]. However, miRNA expression profiling and miRNA targeted genes during saline-alkaline stress in flax have yet to be elucidated. To date, only three reports have been published focusing on flax miRNAs, but they all employed bioinformatics tools to predict flax miRNAs [23-25].

To provide further insights into the role of miRNAs in flax tolerance to saline, alkaline, and saline-alkaline stresses, small RNA and degradome high-throughput sequencing was conducted to analyze samples of flax seedlings grown under alkaline-salt stress (AS2), neutral salt stress (NSS), alkaline stress (AS), and under control conditions (CK). In this study, flax miRNAs, their target genes, functional annotations, and gene expression patterns were revealed under saline, alkaline, and saline-alkaline stresses. These findings should enhance the understanding of regulatory mechanisms involving flax miRNAs expression under saline, alkaline, and saline-alkaline stresses and provide a foundation for future studies to determine the specific functions of these miRNAs. This study is the first report in which small RNA (sRNA) libraries have been constructed and sequenced to identify saline, alkaline, and saline-alkaline tolerance miRNAs in flax.

\section{Result}

Characterization of Lus-miRNAs from deep sequencing of flax sRNA libraries

To identify saline, alkaline, and saline-alkaline responsive miRNAs in flax, four small RNA libraries from flax seedlings treated with AS, AS2, NSS, and water (control) were constructed. Solexa, a high throughput sequencing technology, was employed to sequence these libraries, leading to generation of over 26.5 million clean reads from four libraries. All clean reads were obtained after removal of adapter, insert, and polyA sequences, as well as removal of sequences of RNAs shorter than $18 \mathrm{nt}$ in length (Table 1). Ultimately, over 2.7 million unique sRNAs from four libraries were mapped to the flax genome published in 2012 [21].

The size distribution of all sRNAs was found to be diverse, ranging from $18-30$ nt in length, with the majority measuring 19-25 nt in length (Fig. 1). The sRNAs of $21 \mathrm{nt}$ and $24 \mathrm{nt}$ formed two major classes within the total sRNA. In addition, analysis of the first nucleotide of 18-30 nt sRNAs indicates that many sRNAs possess a uridine (U) at their 5 ' ends. Most of these sRNAs are $21 \mathrm{nt}$ and $22 \mathrm{nt}$ long, with the $21 \mathrm{nt}$ 
Table 1 Summary of data cleaning of MicroRNA-seq

\begin{tabular}{lllllll}
\hline Library & Raw reads & Adaptors removed & Sequences < 18 nt removed & Clean reads & $\begin{array}{l}\text { Total sRNAs mapped } \\
\text { to Genome }\end{array}$ & $\begin{array}{l}\text { Unique sRNAs mapped } \\
\text { to Genome }\end{array}$ \\
\hline CK & $31,675,979$ & 136,110 & 58,807 & $31,309,326$ & $24,393,572$ & $3,486,958$ \\
AS & $29,867,252$ & 65,980 & 105,870 & $29,526,585$ & $19,793,866$ & $3,488,851$ \\
AS2 & $27,379,453$ & 92,632 & 593,274 & $26,536,356$ & $18,749,253$ & $2,767,309$ \\
NSS & $28,449,740$ & 114,587 & 64,740 & $28,105,246$ & $22,212,514$ & $3,058,192$ \\
\hline
\end{tabular}

Abbreviations: AS Alkaline stress, AS2 Alkaline-salt stress, CK Control, Lus Linum usitatissimum, NSS Neutral salt stress

length predominating (Additional file 1). Similar to observations in other plants, most miRNAs here were of 21 and 22 nt in length and possessed a 5' uridine, which is one of the important characteristic features of miRNAs.
High throughput sequencing can be used to verify a large number of known miRNAs and novel specific miRNAs in organisms. From four sRNA libraries in this study, CK, AS, AS2, and NSS libraries, we first identified $118,119,122$, and 120 known Lus-miRNAs, respectively.

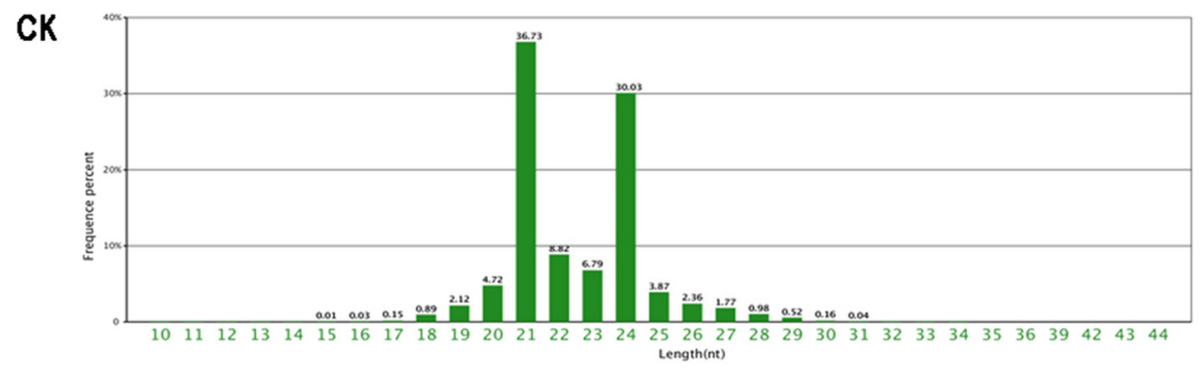

AS

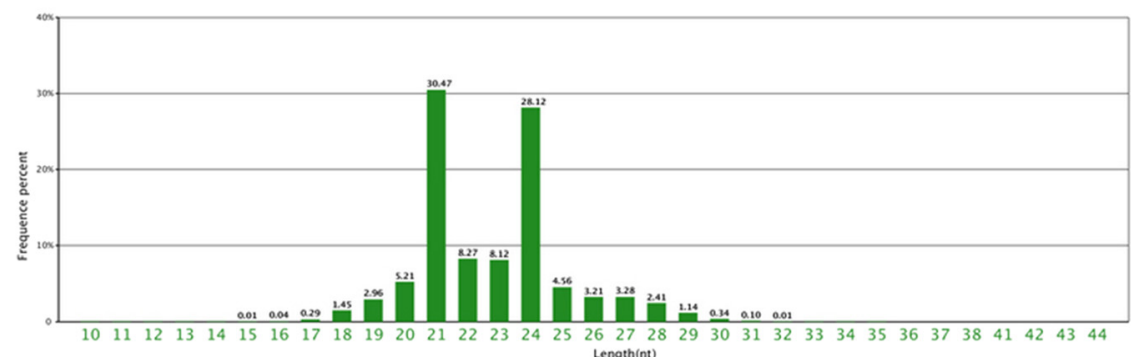

AS2

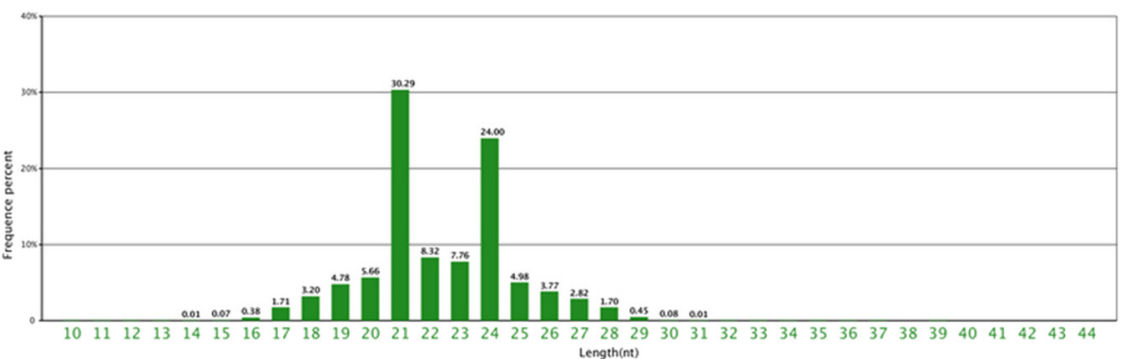

NSS

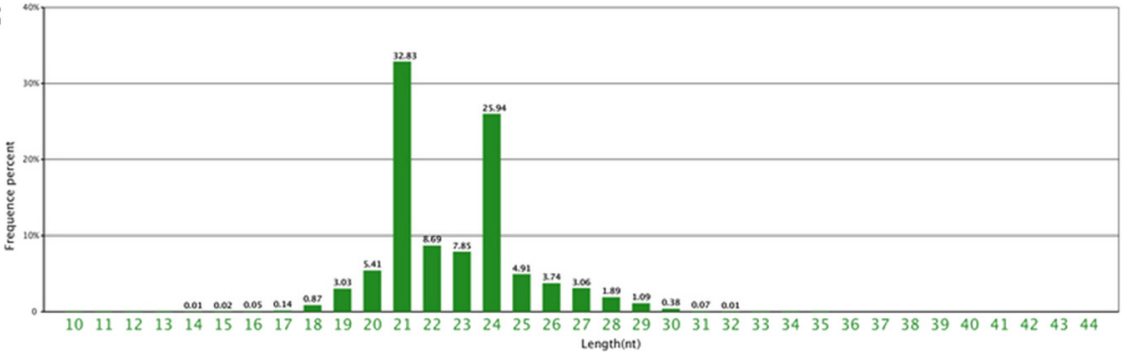

Fig. 1 Summary of the read length distribution of small RNAs. The distributions of the total reads are shown as percentages 
These were assigned to 23 conserved miRNA families after comparing our libraries with known miRNAs from flax and other plant species using miRBase 19.0 (http://www. mirbase.org/). Bioinformatics analysis of the sequencing data, based on the criteria of novel miRNA annotations developed by Meyers [26], resulted in identification of 233, 213, 211, and 212 potential novel Lus-miRNAs in the CK, AS, AS2, and NSS libraries, respectively (Additional file 2).

\section{Discovery of miRNAs responsive to saline, alkaline, and saline-alkaline stresses in flax}

To identify Lus-miRNAs responsive to saline, alkaline, and saline-alkaline stresses, differentially expressed miRNAs in each sample were compared to the control. A false discovery rate (FDR) $<0.001$ and an absolute threshold value of the $\log 2$ ratio fold-change $>1$ were used to determine the statistical significance of relative miRNA abundance values. There were 101, 103, and 101 differentially expressed known miRNAs in the AS, AS2, and NSS libraries, respectively (Additional file 3 ). Of these, 32, 37, and 14 were up-regulated and 69, 66, and 87 were downregulated in the libraries, respectively. Among these, 2, 19, and 13 miRNAs exhibited very high expression differences in their respective libraries, relative to the control (Table 2).

Of particular interest, 66,66 , and 56 novel miRNAs were differentially expressed and of these, 28, 27, and 16 were up-regulated and 38,39 , and 40 were downregulated in the AS, AS2, and NSS libraries, respectively (Additional file 3). Of these, 38, 34, and 34 were highly differentially expressed (Additional file 4). Moreover, several miRNAs were significantly, differentially expressed between two separate libraries. For example, lus-miR398a and lus-miR408a were significantly differentially expressed between AS and AS2, whereas lus-miR160 and lusmiR394 were significantly differentially expressed between AS2 and NSS.

The expression levels of known miRNAs and novel miRNAs in all samples are listed in Additional file 5. The results revealed that two known Lus-miRNAs (lus-miR399f and lus-miR399g) and five novel Lus-miRNAs (novel mir_147, novel_mir_150, novel_mir_27, novel_mir_2, novel_mir_45) were found only in AS, AS2 and NSS, but not in CK, suggesting they were probably induced by saline, alkaline, and saline-alkaline stresses.

\section{The expression patterns of all known and novel miRNAs} under saline, alkaline, and saline-alkaline stresses in flax The expression patterns of all known and novel miRNAs identified were profiled based on their sequencing results. Most of the known and the novel miRNAs showed various degrees of expression under saline, alkaline, and saline-alkaline stresses as compared to the control, with the $\log 2$ values (Treatment/Control) of the fold changes falling between -4 and 4 (Fig. 2, Additional file 3). Of the 23 known miRNA families, 2 (lus-miR408, lus-miR530) and 5 (lus-miR160, lus-miR393, lus-miR394, lus-miR398, lus-miR408) were up-regulated significantly in AS and AS2, respectively. 1 (lus-miR169) and 5 families (lusmiR159, lus-miR160, lus-miR171, lus-miR319, lus-miR394) were down-regulated significantly in AS2 and NSS, respectively (Fig. 2a, Additional file 3). Of the novel miRNAs, 18, 20 , and 12 were significantly up-regulated, while 20,14 , and 12 were significantly down-regulated in AS, AS2, and NSS libraries, respectively (Fig. 2b, Additional file 3). These results suggest that these miRNAs might have coordinating functions in response to saline, alkaline, and saline-alkaline stresses in flax.

Our data also showed that some members of the same miRNA family exhibited different expression patterns under saline, alkaline, and saline-alkaline stresses in flax (Fig. 2). For example, Lus-miR171g is up-regulated in AS but down-regulated in AS2 and NSS, while Lus-miR171i is up-regulated in AS and NSS but down-regulated in AS2. Although these results await further confirmation using other molecular techniques, together they suggest that miRNA members from different families, as well as different members from the same family, may have variable response patterns to saline, alkaline, and salinealkaline stresses.

\section{Prediction and annotation of miRNA target genes}

To further understand the potential functions of the known and novel salt-responsive miRNAs identified in this work, their putative target genes were predicted using the psRNA Target program (http://plantgrn.noble. org/psRNATarget/). 17 differentially expressed known miRNAs and 36 differentially expressed novel miRNAs with high abundance were selected and used to predict putative target genes (Table 2, Additional file 6). Among the 36 novel Lus-miRNAs, 22 had multiple target genes, as exemplified by the novel_mir_231 Lus-miRNA with 261 target genes, indicating these Lus-miRNAs might possess comprehensive functions in flax. Interestingly, while different members of a given miRNA family may target the same genes, even members of diverse miRNA families may also share common target genes. For example, both lus-miR169e and lus-miR169i can target genes encoding jasmonate-zim-domain protein 3 , which indicates that they are functionally conservative members within one family, with similar results observed for lusmiR394a and lus-miR394b. However, members of these distinct families also share a gene target belonging to the FGGY family of carbohydrate kinase. Furthermore, lusmiR159c and lus-miR319a can target the same gene encoding a Myb domain protein, which means their functions may be similar under saline stress in flax (Table 2). 
Table 2 Summary of significant differential expressed genes known miRNA under saline, alkaline, and saline-alkaline stresses

\begin{tabular}{|c|c|c|c|c|c|}
\hline \multirow[t]{2}{*}{ MiR-name } & \multicolumn{3}{|c|}{ Fold-change(log2) } & \multirow[t]{2}{*}{ Target } & \multirow[t]{2}{*}{ Anotation } \\
\hline & $\overline{\mathrm{AS}}$ & ASS & NSS & & \\
\hline lus-miR159c & - & - & $-1.0931792 \downarrow$ & $\begin{array}{l}\text { Lus10036103[a], Lus10016550, Lus10027189, } \\
\text { Lus10017946, Lus10008685[a], Lus10028176, } \\
\text { Lus10013688, Lus10035275, Lus10026142[a], } \\
\text { Lus10009780, Lus10026787[a] }\end{array}$ & $\begin{array}{l}\text { Myb domain protein, Mitochondrial } \\
\text { transcription termination factor family } \\
\text { protein, Transcription regulators }\end{array}$ \\
\hline lus-miR160a/e/f & - & $1.20840613 \uparrow$ & $-3.66411747 \downarrow$ & \multirow{4}{*}{$\begin{array}{l}\text { Lus10024753, Lus10024754, Lus10023519, } \\
\text { Lus10019940, Lus10026510, Lus10016090, } \\
\text { Lus10040403, Lus10009770, Lus10021467 }\end{array}$} & \multirow[t]{4}{*}{ Auxin response factor } \\
\hline lus-miR160b/d & - & $1.20719184 \uparrow$ & $-3.66660396 \downarrow$ & & \\
\hline lus-miR160j & - & $1.20846291 \uparrow$ & & & \\
\hline lus-miR160h/i & - & $1.20203167 \uparrow$ & $-3.69976116 \downarrow$ & & \\
\hline lus-miR169c & - & $-1.13471672 \downarrow$ & - & \multirow[t]{2}{*}{ Lus10017991, Lus10041986, } & \multirow{2}{*}{$\begin{array}{l}\text { Jasmonate-zim-domain protein, GRAS } \\
\text { family transcription factor }\end{array}$} \\
\hline lus-miR169e/i & - & $-1.05752449 \downarrow$ & - & & \\
\hline lus-miR171j & - & - & $-1.02479034 \downarrow$ & $\begin{array}{l}\text { Lus10024029, Lus10041721, Lus10004353, } \\
\text { Lus10028934 }\end{array}$ & GRAS family transcription factor \\
\hline lus-miR319a & - & - & $-1.46989392 \downarrow$ & $\begin{array}{l}\text { Lus10036103[a], Lus10008685[a], } \\
\text { Lus10026142[a], Lus10026787[a] }\end{array}$ & Myb domain protein \\
\hline lus-miR393a/c & - & $1.12123117 \uparrow$ & - & \multirow[t]{2}{*}{ Lus10031991, Lus10035160 } & \multirow[t]{2}{*}{ Auxin signaling F-box } \\
\hline lus-miR393b/d & & $1.00414957 \uparrow$ & - & & \\
\hline lus-miR394a & - & $2.65759041 \uparrow$ & $-3.63085489 \downarrow$ & \multirow{2}{*}{$\begin{array}{l}\text { Lus10000973, Lus10029731, Lus10011354, } \\
\text { Lus10022009, Lus10028656, Lus10028656, } \\
\text { Lus10006975, Lus10015775, Lus10001312, } \\
\text { Lus10003117, Lus10037030, }\end{array}$} & \multirow{2}{*}{$\begin{array}{l}\text { S-adenosyl-L-methionine-dependent } \\
\text { methyltransferases superfamily protein, } \\
\text { Galactose oxidase/kelch repeat superfamily } \\
\text { protein, Signal transduction histidine kinase, } \\
\text { FGGY family of carbohydrate kinase, } \\
\text { Jasmonate-zim-domain protein }\end{array}$} \\
\hline lus-miR394b & - & $2.77702791 \uparrow$ & $-3.82176614 \downarrow$ & & \\
\hline lus-miR398a & $2.51091239 \uparrow$ & $2.69813582 \uparrow$ & - & - & - \\
\hline lus-miR408a & $1.71297796 \uparrow$ & $1.63630636 \uparrow$ & - & Lus10018938, Lus10020012, Lus10028640, Lus10028641 & Plantacyanin, Chloroplast import apparatus \\
\hline
\end{tabular}




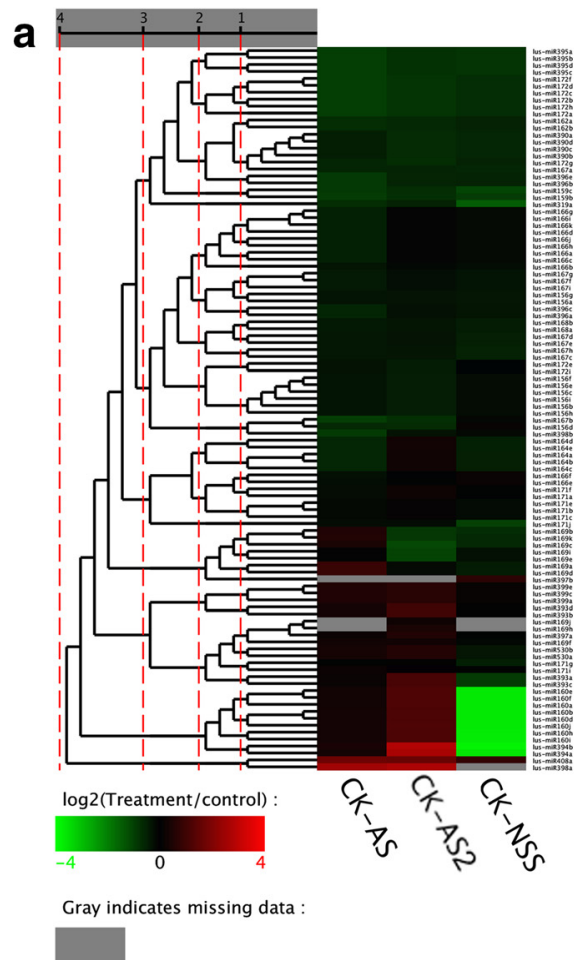

b
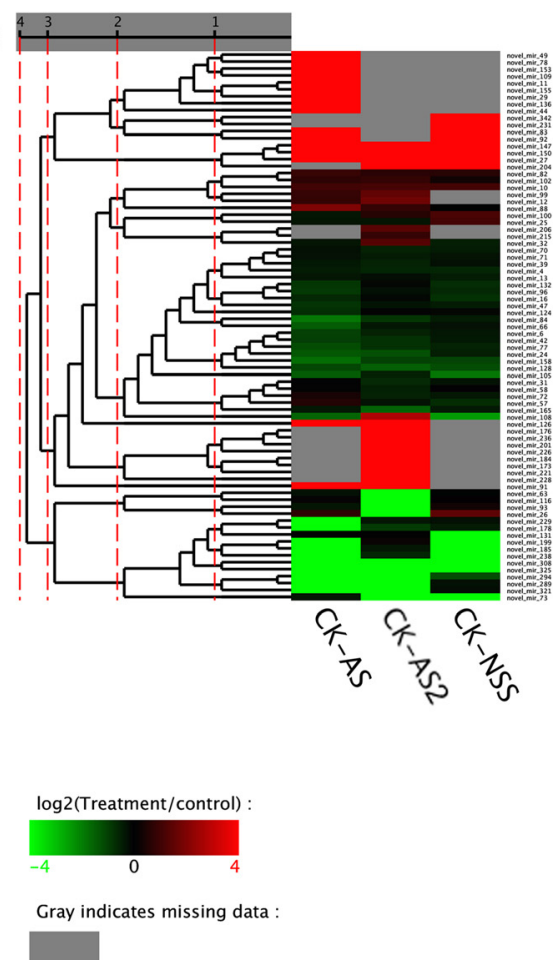

Fig. 2 Cluster analyses of known miRNAs and novel miRNAs. Each line refers to data from one gene. The color bar represents the log 2 RPKM and ranges from green to red. Red indicates that the miRNA has a higher expression level in treated sample; green indicates that the miRNA has higher expression in the control sample and gray indicates that the miRNA has no expression in at least one sample; dotted line indicates that all differentially expressed miRNAs are clustered all in one after four rounds of cluster.

To evaluate the potential functions of these miRNA target genes, GO analysis was used [27]. The miRNA target genes were categorized according to biological process, cellular component, and molecular function (Additional files 7 and 8). The miRNA predicted targets in AS showed enrichment in GO terms in the biological process category, while no enrichment in GO terms was observed in the cellular component and molecular function categories. The results reveal that these target genes possess functions involved in response to stimuli, signaling, catalytic activity, and their expression is significantly altered by saline, alkaline and salinealkaline stresses, in comparison to genes in CK as a whole.

\section{MiRNA targets verified by degradome sequencing}

To further understand the role of miRNA in saline, alkaline and saline-alkaline stresses regulation in flax, degradome sequencing of flax was used to identify miRNA targets (Additional file 9). Although a large number of transcripts exhibited expression changes under saline, alkaline and saline-alkaline stresses, 29 miRNAtarget pairs showed inverse expression pattern changes when the results from miRNA profiling, degradome sequencing, and transcriptome profiling from our previous study were compared (Table 3) [22]. These results indicate that these miRNAs and target genes may play important opposing roles in the response to saline, alkaline and saline-alkaline in flax.

qRT-PCR analysis of miRNAs under saline, alkaline, and saline-alkaline stresses in flax

To confirm the accuracy and reliability of the miRNA-seq results, the same RNA preparation used for Solexa sequencing was used to prepare samples for the qRT-PCR assay. In this study, eight miRNAs (lus-miR156b, lus-miR159c, lus-miR160a, lus-miR168a, lus-miR169a, lus-miR319a, lusmiR393a and lus-miR398a) were randomly selected for analysis of expression levels under saline, alkaline, and saline-alkaline stresses using actin as the internal reference gene (Fig. 3). Results showed that the expression changes of these miRNAs, as determined by qRT-PCR, followed similar trends observed using sequencing results. These results suggest that differentially expressed flax miRNAs had been successfully and accurately identified under saline, alkaline, and saline-alkaline stresses using Solexa sequencing. Of note, the abundance of miR159c, miR168a, and miR319a were lower under saline-alkaline stress.

\section{Discussion}

High throughput sequencing technology has been extensively applied to small RNA research. MiRNAs, as 
Table 3 Complementary expressions between miRNAs and their targets in flax under saline, alkaline, and saline-alkaline stresses

\begin{tabular}{|c|c|c|c|c|c|c|c|c|c|c|c|}
\hline \multirow[t]{2}{*}{ MiRNAs } & \multicolumn{3}{|c|}{ Small RNA sequencing } & \multirow[t]{2}{*}{ Target gene } & \multirow[t]{2}{*}{ Annotation } & \multicolumn{3}{|c|}{ Degradome sequencing } & \multicolumn{3}{|c|}{ DGE sequencing } \\
\hline & AS & AS2 & NSS & & & Position & Lignment score & Category & AS & AS2 & NSS \\
\hline lus-miR156g/a & Down & Down & Down & Lus10000257 & Tetratricopeptide repeat (TPR)-like superfamily protein & 761 & 4.5 & 4 & Up & Up & Up \\
\hline lus-miR159b & Down & Down & Down & Lus10010495 & Cystatin/monellin superfamily protein & 335 & 4.5 & 3 & Up & Up & Up \\
\hline lus-miR160a/b/d/e/f/h/i/j & Up & Up & Down & Lus10041268 & Transducin/WD40 repeat-like superfamily protein & 1014 & 4 & 2 & Down & Down & Up \\
\hline lus-miR162a/b & Down & Down & Down & Lus10015483 & Heat shock protein 70 ( Hsp 70) family protein & 1243 & 4.5 & 2 & Up & Up & Up \\
\hline lus-miR164a/b/c/d/e & Down & Up & Down & Lus10006635 & ARM repeat superfamily protein & 153 & 4 & 4 & Up & Down & Up \\
\hline lus-miR166a/c/d/g/h/j & Down & Down & Down & Lus10020493 & Pathogenesis-related gene 1 & 220 & 4 & 2 & Up & Up & Up \\
\hline lus-miR167a & Down & Down & Down & Lus10014324 & G-box binding factor 1 & 679 & 3.5 & 2 & Up & Up & Up \\
\hline lus-miR168a/b & Down & Down & Down & Lus10000074 & Methionine gamma-lyase & 469 & 4.5 & 4 & Up & Up & Up \\
\hline lus-miR169a/d & Up & Down & Down & Lus10014674 & Transducin/WD40 repeat-like superfamily protein & 638 & 4 & 4 & Down & Up & Up \\
\hline lus-miR169e/i & Down & Down & Down & Lus10006846 & Profilin 5 & 96 & 4.5 & 4 & Up & Up & Up \\
\hline lus-miR169g/l & Up & Down & Up & Lus10030904 & Alpha/beta-Hydrolases superfamily protein & 755 & 4.5 & 4 & Down & Up & Down \\
\hline lus-miR171b/c/e & Down & Down & Down & Lus10009876 & UDP-glucosyl transferase 85A3 & 952 & 4 & 4 & Up & Up & Up \\
\hline lus-miR171d & Up & Down & Down & Lus10017991 & Jasmonate-zim-domain protein 3 & 594 & 4.5 & 2 & Down & Up & Up \\
\hline lus-miR172a/b/c/d/f/h & Down & Down & Down & Lus10001322 & Deoxyxylulose-5-phosphate synthase & 1796 & 4 & 4 & Up & Up & Up \\
\hline lus-miR319b & Down & Down & Down & Lus10009442 & O-methyltransferase family protein & 502 & 4 & 4 & Up & Up & Up \\
\hline lus-miR390a/b/c/d & Down & Down & Down & Lus10015906 & Purine permease 3 & 857 & 4.5 & 4 & Up & Up & Up \\
\hline lus-miR393a/b/c/d & Up & Up & Down & Lus10040438 & F-box family protein & 1620 & 3.5 & 4 & Down & Down & Up \\
\hline lus-miR394a/b & Up & Up & Down & Lus10018337 & Pyruvate dehydrogenase kinase & 536 & 4.5 & 2 & Down & Down & Up \\
\hline lus-miR395a/b/c/d & Down & Down & Down & Lus10006629 & ATP sulfurylase 1 & 339 & 2.5 & 0 & Up & Up & Up \\
\hline lus-miR396a/c & Down & Down & Down & Lus10001321 & Xylose isomerase family protein & 648 & 3.5 & 2 & Up & Up & Up \\
\hline lus-miR397a & Up & Up & Down & Lus10004434 & REF4-related 1 & 1875 & 4.5 & 4 & Down & Down & Up \\
\hline lus-miR397b & Down & Up & Up & Lus10001002 & 3-deoxy-d-arabino-heptulosonate 7-phosphate synthase & 581 & 4.5 & 4 & Up & Down & Down \\
\hline lus-miR398a & Up & Up & Up & Lus10016155 & Copper/zinc superoxide dismutase 2 & 449 & 4 & 0 & Down & Down & Down \\
\hline lus-miR398b/c/d/e & Down & Down & Down & Lus10003315 & Myosin family protein with Dil domain & 4406 & 4.5 & 2 & Up & Up & Up \\
\hline lus-miR399b/d & Down & Up & Down & Lus10019360 & Trigalactosyldiacylglycerol2 & 323 & 4.5 & 4 & Up & Down & Up \\
\hline lus-miR399f/g & Up & Up & Up & Lus10003060 & Cofactor-independent phosphoglycerate mutase & 1097 & 4 & 4 & Down & Down & Down \\
\hline lus-miR408a & Up & Up & Up & Lus10003138 & Cyclophilin 20-2 & 860 & 4.5 & 2 & Down & Down & Down \\
\hline lus-miR530a/b & Up & Up & Down & Lus10001902 & WRKY family transcription factor & 490 & 4.5 & 2 & Down & Down & Up \\
\hline lus-miR828a & Down & Up & Up & Lus10013640 & Ribosomal protein L3 family protein & 570 & 4.5 & 4 & Up & Down & Down \\
\hline
\end{tabular}



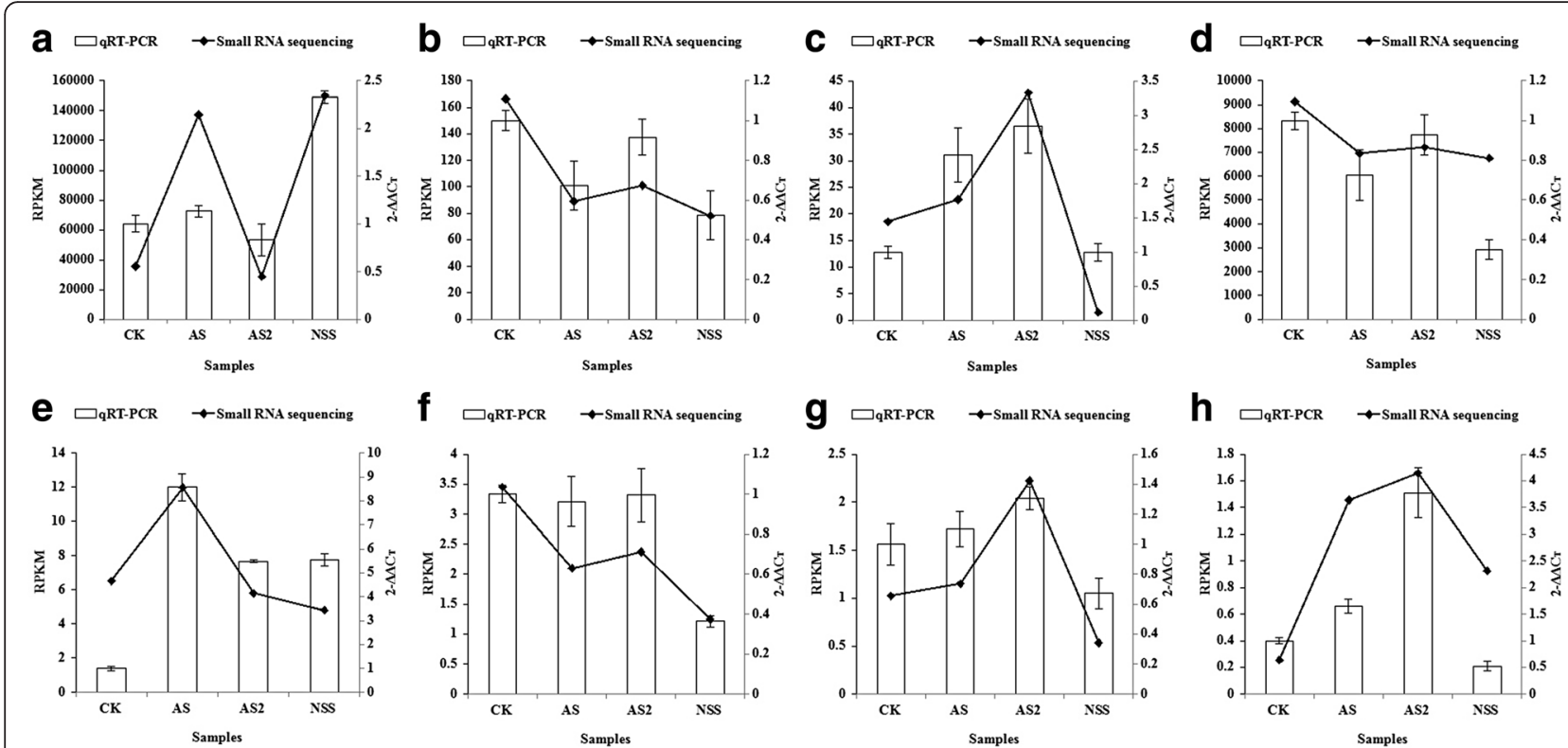

Fig. 3 Comparison of the miRNA expression profiles determined by quantitative real-time RT-PCR (qRT-PCR) and deep sequencing. Bars represent the standard deviations of three replicates. a lus-miR156b; b lus-miR159c; c lus-miR160a; d lus-miR168a; e lus-miR169a; f lus-miR319a; g lusmiR393a; $\mathbf{h}$ lus-miR398a

regulators of target genes, have been reported to play major roles in a plant's response to abiotic stresses, including dehydration, freezing, salinity, and alkalinity [28]. Many miRNAs involved in the high-salinity stress response in plants have been identified [29, 30]; however, little research on a genome-wide scale has focused on the saline, alkaline, and saline-alkaline responsive miRNAs in flax. In the present study, miRNAs were identified and characterized from flax under saline, alkaline, and saline-alkaline stresess using experimental characterization of sRNAs. This work will provide new information to facilitate further research into the functions, biological pathways, and evolution of flax sRNA and its target genes.

In this study, we constructed four sRNA cDNA libraries from flax seedlings treated with AS, AS2, NSS, and CK. Subsequently, 124 known miRNAs belonging to 23 conserved miRNA families and 394 novel miRNAs were identified after sequencing and analysis of the sRNAs of flax. Analysis of the predicted targets of the miRNAs using the GO and KEGG databases indicated that a range of metabolic pathways and biological processes known to be associated with salt stress were upregulated in flax treated with salt. Most of the miRNAs that were obtained in our library have a $5^{\prime}-\mathrm{U}$, as has been reported in other plants, which is in accordance with the known structures of the mature miRNAs [31]. The results indicated the presence of a range of sRNAs, of lengths 14-32 nt in flax, with most of the unique sequence reads of $24 \mathrm{nt}$ in length with $21 \mathrm{nt}$ length reads next in predominance. This observation is in agreement with previous reports for grapevine and soybean [32, 33], as well as with results indicating that the most common sRNAs in celery and maize were $24 \mathrm{nt}$ in length [34,35]. However, these results differ from results reported for Chinese cabbage and foxtail millet. Some plant species, including Arabidopsis thaliana, had been shown to contain substantially more $24 \mathrm{nt}$ sRNAs than $21 \mathrm{nt}$ sRNAs [36]; on the other hand, sRNAs populations with more members of length $21 \mathrm{nt}$ than $24 \mathrm{nt}$ were reported in Brassica juncea and in Japanese apricot with imperfect flower buds $[37,38]$. Taken together, all of these results suggest that some differences might exist in sRNA biogenesis pathways between various plant species.

Many miRNAs with a wide range of expression levels were found in the AS, AS2, NSS, and CK libraries. The most abundantly expressed miRNA family across the four libraries was miR156, specifically including miR156b, miR156c, miR156e, miR156f, miR156h, and miR156i, as was also observed in Leymus chinensis (Additional file 6) [39]. Some miRNAs were differentially expressed between the stress libraries and control library (Additional file 3). There were 2, 19, and 13 highly differentially expressed miRNAs in AS, ASS, and NSS, as compared to CK, respectively. Two miRNAs (lus-miR398a and lus-miR408a) were greatly up-regulated in AS and AS2 as compared to CK, in opposition to the results of pea plants subjected to drought stress [40]. Expression of ten miRNAs (lus-miR160a, lus-miR160b, lus-miR160d, lus-miR160e, lus-miR160f, lus-miR160h, lus-miR160i, lus-miR160j, 
lus-miR394a and lus-miR394b) were significantly altered in AS2 and NSS as compared to CK, however, these miRNAs were significantly up-regulated in AS2 and significantly down-regulated in NSS. The results indicated that these lus-miRNAs exhibit different functions in response to AS2 and NSS in flax. In agreement with our results, previous studies have consistently demonstrated that miR394 was responsive to stress conditions, including salt and drought stress [41]. However, miR160 only previously had been reported to play an important role in plant development, not in stress responses, as shown in this work [42].

Our libraries have facilitated the identification of a large number of conserved saline, alkaline, and salinealkaline responsive lus-miRNAs, including lus-miR156, lusmiR159, lus-miR160, lus-miR162, lus-miR164, lus-miR166, lus-miR167, lus-miR168, lus-miR169, lus-miR171, lusmiR172, lus-miR319, lus-miR390, lus-miR393, lus-miR394, lus-miR395, lus-miR396, lus-miR397, lus-miR398, lusmiR399, lus-miR408, lus-miR503, and lus-miR828, some of which were confirmed here using qRT-PCR (Fig. 3). Several differentially regulated miRNAs have been identified in saltstressed plants. Our results agree with results in a previous study involving Arabidopsis thaliana [43], Zea mays [44], Vigna unguiculata [45], Populus trichocarpa [46], Populus tremula [13], Oryza sativa [47, 48], in which 22 saltresponsive miRNAs (miR156, miR159, miR160, miR162, miR164, miR166, miR167, miR168, miR169, miR170/ miR 171, miR172, miR319, miR390, miR393, miR394, miR395, miR396, miR397, miR398, miR399, miR408 and miR530) were reported to be involved in the high salinity stress response (Table 4). In Arabidopsis thaliana, miR156, miR159, miR167, miR168, miR171, miR319, miR393, miR394, miR396, and miR397 were all upregulated in response to salt stress, whereas miR398 was down-regulated [14, 43]. Furthermore, miR169g and miR169n were also reported to be induced by high salinity [49]. Recently, a study of maize roots using miRNA microarray hybridization indicated that members of the miR156, miR164, miR167, and miR396 families were down-regulated by salt shock, whereas miR162 and miR168 were up-regulated [44]. The expression of miR389, miR400, miR402, miR403, and miR407a were inhibited by salt, cold, dehydration, and abscisic acid (ABA) in Arabidopsis [14], while these miRNAs and their variants were not detected in flax.

In this study, target genes for miRNAs that were differentially expressed in the four libraries were identified by searching for corresponding plant miRNA target sites, which are predominantly located in open reading frames. Many antioxidant enzyme and transcription factors have been predicted to be targets of conserved, flaxspecific miRNAs (Table 3). Some of these proteins have been well-studied and their roles in salt tolerance or the
Table 4 MicroRNAs responsive to neutral saline stress $(\mathrm{NaCl})$ in diverse plant species

\begin{tabular}{|c|c|c|}
\hline MiR-name & Plant species & Refs \\
\hline miR156 & Lus $\uparrow \downarrow \downarrow, Z m a \downarrow, A t h \uparrow, ~ V u n \uparrow$ & $43,44,49$ \\
\hline miR159 & Lus $\downarrow^{a}$, Ath $\uparrow$, Osa $\downarrow$ & 43,48 \\
\hline $\operatorname{miR} 160$ & Lus $\downarrow^{a}$, Osa $\downarrow$, Vun $\uparrow$ & 43,48 \\
\hline miR162 & 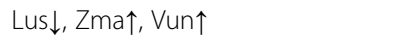 & 43,44 \\
\hline miR164 & Lus $\downarrow, Z m a \downarrow$ & 44 \\
\hline miR166 & Lus $₫ \downarrow$ & \\
\hline miR167 & Lus $\downarrow, Z m a \downarrow$, Ath $\uparrow$ & 43,44 \\
\hline miR168 & Lus $\downarrow$, Zma $\uparrow$, Ath $\uparrow$, Pte $\uparrow$, Vun $\uparrow$ & $13,16,44,49$ \\
\hline miR169 & Lus $\downarrow$, Zma $\uparrow$, Ath $\uparrow$, Pte $\downarrow$, Osa $\uparrow$, Vun $\uparrow$ & $13,16,43,49$ \\
\hline miR170/miR171 & Lus $\uparrow \& \downarrow^{a}$, Ath $\uparrow, P t c \downarrow$ & 43,52 \\
\hline miR172 & Lus $\& \downarrow$ & \\
\hline miR319 & Lus $\downarrow^{a}$, Ath $\uparrow$, Osa $\downarrow$ & 43,48 \\
\hline miR390 & Lus $\downarrow$ & \\
\hline miR393 & Lus $\downarrow$, Ath $\uparrow$, Ptc $\uparrow$, Osa $\downarrow$ & 43,49 \\
\hline miR394 & Lus $\downarrow^{a}$, Ath $\uparrow$, Osa $\downarrow$ & 43,48 \\
\hline miR395 & 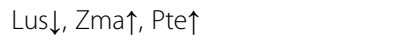 & 13,44 \\
\hline miR396 & Lus $\downarrow$, Zma $\downarrow$, Ath $\uparrow$, Osa $\downarrow$ & $43,44,49$ \\
\hline miR397 & Lus $\uparrow$, Ath $\uparrow$ & 14 \\
\hline miR398 & Lus $\downarrow$, Ath $\downarrow$, Pte $\uparrow$ & 13,43 \\
\hline miR399 & Lus $\uparrow$, Pte $\uparrow$ & 13 \\
\hline miR408 & 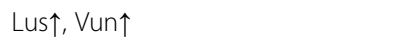 & 49 \\
\hline $\operatorname{miR530}$ & Lus $\downarrow$, Ptc $\downarrow$, Osa $\downarrow$ & 48,52 \\
\hline
\end{tabular}

Abbreviations: Ath Arabidopsis thaliana, Lus Linum usitatissimum, MiR MicroRNA, Ptc Populus trichocarpa, Pte Populus tremula, Osa Oryza sativa, Vun Vigna unguiculata; Zma Zea mays, $\uparrow$, Upregulated; $\downarrow$, Downregulated; $\uparrow \& \downarrow$, Some members were upregulated, and some were downregulated

${ }^{a}$ Significant differential expressed known miRNA in flax

stress response have been established. Previous studies have demonstrated that miR398 family members are associated with high salt stress [13]. From our target prediction analysis, the miR398a-targeted gene codes for a copper/zinc superoxide dismutase (CuZnSOD, EC 1.15.1.1), known to be important scavengers of reactive oxygen species (ROS) to protect cells from damage. Recent studies have also demonstrated that this protein plays significant roles in salt stress response pathways. These results are in agreement with our data for miR398 [50, 51], suggesting significant involvement of this miRNA and its target in the salt stress response in plants.

In this work, the salt-responsive miR530 has been shown to explicitly target WRKY family transcription factors (TFs). This is in agreement with previous findings showing that plant-specific WRKY TFs are involved in stress responses such as cold, high salinity or drought, as well as in abscisic acid signaling. A parallel study reported that WRKY TFs act in response to salt stress in many plants, including rice [52], maize [53], and cotton 
[54]. However, only one paper has focused on miR530, demonstrating that the target gene of miR530 was KNAT [55], which regulates inflorescence architecture in Arabidopsis [56]. Therefore, the relationship between miR530 and salt stress should be given more attention. Meanwhile, there are several miRNAs without identified target genes; these results could be the result of inaccurate target predictions, or these might be low-abundance miRNAs with limited or no activity. It is also possible that miRNAs might exist that have no targets. Nevertheless, the $\mathrm{KO}$ and $\mathrm{GO}$ analyses revealed that many of the genes targeted by miRNAs in flax are related to salt stress, supporting the hypothesis that miRNAs play an important role in the response of flax to salinity. Greater understanding of these miRNAs and their targets should facilitate future development of flax with greater resistance to salt stress.

\section{Conclusions}

Four small RNA libraries and one degradome library were constructed under saline, alkaline, and saline-alkaline stresses in flax. By using high-throughput sequencing, the miRNAs profile of flax was investigated to illustrate the miRNAs expression differences among AS2, NSS and AS. Many known Lus-miRNAs and potential novel Lus-miRNAs were identified in the CK, AS, AS2, NSS libraries, respectively. After assessment of differential expression profiles, 17 known Lus-miRNAs and 36 novel Lus-miRNAs were selected and used to predict putative target genes. Gene ontology term enrichment analysis revealed target genes that were involved in responses to stimuli, including signaling and catalytic activity. Eight Lus-miRNAs were selected for analysis using qRT-PCR to confirm the accuracy and reliability of the miRNA-seq results. Degradome sequencing and transcriptome profiling showed that expression of 29 miRNA-target pairs displayed inverse expression patterns under saline, alkaline, and saline-alkaline stresses. Identification and characterization of flax miRNAs, their target genes, functional annotations, and gene expression patterns are reported in this work. These findings will enhance our understanding of flax miRNA regulatory mechanisms under saline, alkaline, and saline-alkaline stresses and provide a foundation for future elucidation of the specific functions of these miRNAs.

\section{Methods}

\section{Plant materials and stress treatments}

The fiber flax plant cultivar used in this study, Heiya No. 19, was obtained from the Industrial Crops Institute, Heilongjiang Academy of Agricultural Sciences (Harbin, P.R.China). Flax seeds were grown on sterilized vermiculite in cups. All plants were cultivated in climate chambers at $22{ }^{\circ} \mathrm{C}$ day $/ 18{ }^{\circ} \mathrm{C}$ night with a $16 \mathrm{~h}$ day $/ 8 \mathrm{~h}$ night photoperiod cycle, $70 \%$ relative humidity, and a light intensity of $3000 \mathrm{~lx}$. The plants were irrigated with onehalf strength Murashige and Skoog medium every 3 days.

The stress treatment was the same as previously described [22]. For the treatments, the 3-week-old seedlings showing appropriate growth states were exposed to alkaline-salt stress (AS2, $25 \mathrm{mM} \mathrm{Na}_{2} \mathrm{CO}_{3}, \mathrm{pH}$ 11.6), neutral salt stress (NSS, $50 \mathrm{mM} \mathrm{NaCl}$ ), and alkaline stress (AS, $\mathrm{NaOH}$, $\mathrm{pH}$ 11.6), respectively. In parallel, the same numbers of seedlings were transferred to distilled water as a control (CK). After exposing the seedlings to stress solutions for $18 \mathrm{~h}$, whole seedlings were harvested, frozen immediately in liquid nitrogen, and stored at $-80{ }^{\circ} \mathrm{C}$ before use. The control plants were also harvested and frozen at the same time. There were more than ten seedlings in each sample.

\section{Construction and sequencing of small RNA libraries}

Total RNA was extracted from flax using Trizol Reagent (Invitrogen, USA), following the manufacturer's instructions. Total RNA quantity and purity were assayed with the NanoDrop 2000 spectrophotometer (Thermo Scientific, USA) at 260/280 $\mathrm{nm}$ (ratios were between 1.8 and 2.0). After assessing RNA integrity using $2 \%$ agarose gel electrophoresis, four sRNA libraries were constructed using RNA extracted from the four different treatments. The four libraries were sequenced using Solexa sequencing (Illumina, USA) at the Beijing Genomics Institute (BGI, Shenzhen, China).

\section{Bioinformatic analysis of miRNAs}

The 49 nt sequence reads from HiSeq sequencing were subjected to data cleaning analysis to remove low quality sequence tags and 5' adaptor contaminants from the reads, leaving clean reads for subsequent analysis. Next, the length distribution of the clean reads for the various samples was summarized. Next, alignment of small RNAs to the miRNAs precursor of the corresponding flax species was performed using miRBase to obtain the miRNAs count using the following detailed criteria. First, high stringency alignment of reads to the miRNAs precursor was performed in miRBase with no mismatches. Second, based on the first criteria, the reads were next aligned to the mature miRNAs in miRBase with at least a 16 nt overlap allowing offsets. Those miRNAs satisfying both criteria were counted to measure the expression of identified miRNAs and were next analyzed to determine the base bias for the first position of identified miRNAs of certain lengths and the base bias for each position of all identified miRNAs, respectively. The novel miRNA was de novo identified by mapping to the genome and predicting loci using Mireap.

To identify differences in miRNAs expression levels under salt stress, the number of reads for each identified 
miRNA was normalized against the total number of reads in the corresponding library. Comparison of the known miRNAs expression between two samples allowed identification of differentially expressed miRNAs. Comparison of the expression of miRNAs between two samples was visualized by plotting a Log2-ratio figure showing clustering of miRNAs with similar expression patterns in both samples.

\section{Prediction of miRNA targets}

After identifying miRNAs with expression patterns that reflect responses to salt stress, putative targets of known and novel miRNAs were predicted using psRNATarget (http://plantgrn.noble.org/psRNATarget/) with default parameters using flax genome settings (http://phytozome. jgi.doe.gov/pz/portal.html). MiRNA targets were next further validated using degradome sequencing. To further investigate the biological functions of miRNAs in flax, the predicted target genes were used to annotate their functions and pathways using the Gene Ontology database (GO, http://www.geneontology.org/). The GO terms corresponding to the target genes were annotated according to their biological processes, molecular functions or involvement as cellular components using Blast2GO.

\section{Degradome sequencing sampling/procedures}

By using Illumina Hiseq 2000 sequencing system, degradome sequencing takes SE50 sequencing strategy and produces 49 nt raw reads. The 3' adaptor will be trimmed before bioinformatics analysis to get real degradome fragments whose length between 20 and 21 nt. After preprocssing, clean tags are generated and stored. Classify clean tags by the alignment to database and remove the ncRNAs. At last, identify the miRNA-mRNA pairs by mapping to reference genes.

The tags mapped to CDNA_sense were used to predict cleavage sites. The specific sites at which a miRNA will cleave gene. Cleavage sites at different positions of one gene are different cleavage sites. The specific miRNA-mRNA pair at cleavage site. Cleavages induced by different miRNAs sequences are different cleavage events, no matter if they are at the same cleavage site. The height of the degradome peak at each occupied transcript position is placed into one of five possible categories: $0,>1$ raw reads at the position, abundance at position is equal to the maximum on the transcript, and there is only one maximum on the transcript; $1,>1$ raw reads at the position, abundance at position is equal to the maximum on the transcript, and there is more than one maximum position on the transcript; 2, >1 raw reads at the position, abundance at position is less than maximum but higher than the median for the transcript; $3,>1$ raw reads at the position, abundance at position is equal to or less than the median for the transcript; 4, Only 1 raw read at the position.

\section{Detection of miRNA expression using qRT-PCR to validate} sequencing results

To validate the results from the bioinformatics-based analysis, stem-loop real-time quantitative RT-PCR was performed. The qRT-PCR primers were designed from miRNA sequences determined above and the reverse transcription was carried out using the RNA from the same four flax libraries (Additional file 10). qRT-PCR was performed using an ABI 7500 Real-Time PCR System (Applied Biosystems, USA) using SYBR Green I (TOYOBO) with the following program: $94{ }^{\circ} \mathrm{C}$ for 30 s, followed by 40 cycles of $94{ }^{\circ} \mathrm{C}$ for $15 \mathrm{~s}, 60{ }^{\circ} \mathrm{C}$ for $15 \mathrm{~s}$, and $72{ }^{\circ} \mathrm{C}$ for $45 \mathrm{~s}$. At the end of PCR reaction, a melting curve was determined. All reactions were conducted at least three times using U6 as an internal control. Three technical replicates were used for qRT-PCR. The relative expression of the miRNA was calculated using the $2^{-\Delta \Delta \mathrm{Ct}}$ method. Statistical tests for qRT-PCR comparisons and small RNA seq was performed by dual axis mapping method of Excel.

\section{Additional files}

Additional file 1: First nucleotide bias of 18-30 nt sRNA tags. (TIF $4533 \mathrm{~kb}$ ) Additional file 2: Summary of known miRNA and novel miRNA in each sample. (XLS $19 \mathrm{~kb}$ )

Additional file 3: Summary of intersample differential expression known miRNA and novel miRNA. (XLS 92 kb)

Additional file 4: Summary of significant differential expressed novel miRNA. (XLS $32 \mathrm{~kb}$ )

Additional file 5: Summary of known miRNA and novel miRNA expression level in all samples. (XLS $81 \mathrm{~kb}$ )

Additional file 6: Identified targets of significant differential expressed novel miRNAs in flax. (XLS $22 \mathrm{~kb}$ )

Additional file 7: GO ontology statistics of target genes of differential expressed known miRNAs and novel miRNAs. (PDF 275 kb)

Additional file 8: GO ontology statistics of targets of known miRNAs and novel miRNAs having differential expression. (XLS $20 \mathrm{~kb}$ )

Additional file 9: Summary of data cleaning of degradome sequencing. (XLS $58 \mathrm{~kb}$ )

Additional file 10: The primers used in this study. (XLS 8 kb)

\section{Abbreviations}

ABA: abscisic acid; AS: alkaline stress; AS2: alkaline-salt stress; BGI: Beijing Genomics Institute; CK: control; CuZnSOD: copper/zinc superoxide dismutase; FDR: false discovery rate; GO: gene ontology; KEGG: kyoto encyclopedia of genes and genomes; KO: KEGG orthology; Lus: Linum usitatissimum; Mir: microRNA; MiRNA: microRNA; NCBI: National Center for Biotechnology Information; NSS: neutral salt stress; qRT-PCR: quantitative RT-PCR; SRA: short read archive; sRNA: small RNA; TFs: transcription factors; U: uridine;

UV: ultraviolet.

\section{Acknowledgements}

We thank the Institute of Industrial Crops, Heilongjiang Academy of Agricultural Sciences for providing the flax seeds.

\section{Authors' contributions}

YY, FG and JZ designed the experiments and drafted the manuscript. YY, HC, $H Y, L C$ and LZ performed the RNA extraction and high-throughput sequencing 
data analysis. YY, DZ, WH, GW and SZ prepared plant materials and carried out qRT-PCR analysis. All authors read and approved the final manuscript.

\section{Availability of data and materials}

All of the short reads obtained in this work were deposited in the National Center for Biotechnology Information (NCBI) and can be accessed in the Short Read Archive (SRA) under the accession number (PRJNA305953).

\section{Funding}

This work was supported by grants from the Heilongjiang Postdoctoral Funding (LRB 127555), Doctoral Scientific Research Foundation of Heilongjiang Academy of Agricultural Sciences (201507-41), National Hemp Industry Technology System (CARS-19) and Project of Harbin Innovative Talents (2013RFQYJ162).

\section{Consent to publish}

Not applicable.

\section{Competing interests}

The authors declare that they have no competing interests.

\section{Ethics (and consent to participate)}

Not applicable.

\section{Author details}

${ }^{1}$ Heilongjiang Academy of Agricultural Sciences Postdoctoral Programme, Harbin 150086, People's Republic of China. ${ }^{2}$ Institute of Industrial Crops, Heilongjiang Academy of Agricultural Sciences, Harbin 150086, People's Republic of China. ${ }^{3}$ Division of Insect-borne Parastitic Disease Control and Prevention, Harbin Center for Disease Control and Prevention, Harbin 150056, People's Republic of China. ${ }^{4}$ Alberta Innovates Technology Futures, Vegreville, Alberta T9C 1 T4, Canada.

\section{Received: 10 January 2016 Accepted: 17 May 2016}

Published online: 27 May 2016

\section{References}

1. Zhu JK. Salt and drought stress signal transduction in plants. Annu Rev Plant Biol. 2002;53(4):247-73.

2. Jin H, Plaha P, Parka JY, Hong CP, Lee IS, Yang ZH, Jiang GB, Kwak SS, Liu SK, Lee JS, Kim YA, Lim YP. Comparative EST profiles of leaf and root of leymus chinensis, a xerophilous grass adapted to high ph sodic soil. Plant Sci. 2006;170(6):1081-6.

3. Voinnet O. Origin, biogenesis, and activity of plant microRNAs. Cell. 2009;136(4):669-87.

4. Lee RC, Feinbaum RL, Ambros V. The c. elegans heterochronic gene lin-4 encodes small RNAs with antisense complementarity to lin-14. Cell. 1993;75(5):843-54.

5. Reinhart BJ, Weinstein EG, Rhoades MW, Bonnie B, Bartel DP. MicroRNAs in plants. Genes Dev. 2002;16(13):1616-26.

6. Tang ZH, Zhang LP, Xu CG, Yuan SH, Zhang FT, Zheng YL, Zheng YL, Zhao $\mathrm{CP}$. Uncovering small RNA-mediated responses to cold stress in a wheat thermosensitive genic male-sterile line by deep sequencing. Plant Physiol. 2012;159(2):721-38

7. Wang $T$, Chen L, Zhao M, Tian Q, Zhang WH. Identification of droughtresponsive microRNAs in Medicago truncatula by genome-wide highthroughput sequencing. BMC Genomics. 2011;12(36):367.

8. Covarrubias AA, Reyes JL. Post-transcriptional gene regulation of salinity and drought responses by plant microRNAs. Plant Cell Environ. 2010;33(4):481-9.

9. Sunkar R, Kapoor A, Zhu J. Posttranscriptional induction of two Cu/Zn superoxide dismutase genes in Arabidopsis is mediated by downregulation of mir398 and important for oxidative stress tolerance. Plant Cell. 2006;18(8):2051-65

10. Licausi F, Weits DA, Pant BD, Scheible WR, Geigenberger P, van Dongen JT. Hypoxia responsive gene expression is mediated by various subsets of transcription factors and miRNAs that are determined by the actual oxygen availability. New Phytol. 2011;190(2):442-56.

11. Jia X, Ren L, Chen Q, Li R, Tang G. UV-B-responsive microRNAs in Populus tremula. J Plant Physiol. 2009;166(18):2046-57.
12. Chen $L$, Wang TZ, Zhao MG, Tian QY, Zhang WH. Identification of aluminum-responsive microRNAs in Medicago truncatula by genome-wide high-throughput sequencing. Planta. 2012;235(2):375-86.

13. Jia X, Wang WX, Ren L, Chen QJ, Mendu V, Willcut B, Dinkins R, Tang X, Tang G. Differential and dynamic regulation of miR398 in response to ABA and salt stress in Populus tremula and Arabidopsis thaliana. Plant Mol Biol. 2009;71(1-2):51-9.

14. Sunkar R, Zhu JK. Novel and stress-regulated microRNAs and other small RNAs from Arabidopsis. Plant Cell. 2004;16(8):2001-19.

15. Jagadeeswaran G, Saini A, Sunkar R. Biotic and abiotic stress down-regulate miR398 expression in Arabidopsis. Planta. 2009;229(4):1009-14.

16. Zhao BT, Ge LF, Liang RQ, Li W, Ruan K, Lin HX, Jin YX. Members of miR-169 family are induced by high salinity and transiently inhibit the NF-YA transcription factor. BMC Mol Biol. 2009;10:29.

17. Gao P, Bai X, Yang L, Lv DK, Pan X, Li Y, Cai H, Ji W, Chen Q, Zhu YM. OsaMIR393: a salinity- and alkaline stress-related microRNA gene. Mol Biol Rep. 2011:38(1):237-342.

18. Gao P, Bai X, Yang L, Lv DK, Li Y, Cai H, Ji W, Guo DJ, Zhu YM. Overexpression of osa-MIR396c decreases salt and alkali stress tolerance. Planta. 2010;231(5):991-1001.

19. McHughen A. Salt tolerance through increased vigor in a flax line (STS-II) selected for salt tolerance in vitro. Theor Appl Genet. 1987;74:727-32.

20. El-Beltagi HS. Some biochemical markers for evaluation of flax cultivars under salt stress conditions. Nat Fibers. 2008;5(4):316-30.

21. Wang Z, Hobson N, Galindo L, Zhu S, Shi D, McDill J, Yang L, Hawkins S, Neutelings G, Datla R, Lambert G, Galbraith DW, Grassa CJ, Geraldes A, Cronk QC, Cullis C, Dash PK, Kumar PA, Cloutier S, Sharpe AG, Wong GK, Wang J, Deyholos MK. The genome of flax (Linum usitatissimum) assembled de novo from short shotgun sequence reads. Plant J. 2012;72: 461-73.

22. Yu Y, Huang WG, Chen HY, Wu GW, Yuan HM, Song XX, Kang QH, Zhao DS, Jiang WD, Liu Y, Wu JZ, Cheng LL, Yao YB, Guan FZ. Identification of differentially expressed genes in flax (Linum usitatissimum L.) under salinealkaline stress by digital gene expression. Gene. 2014;549(1):113-22.

23. Barvkar VT, Pardeshi VC, Kale SM, Qiu SQ, Rollins M, Datla R, Gupta VS, Kadoo NY. Genome-wide identification and characterization of microRNA genes and their targets in flax (linum usitatissimum). Planta. 2013;237(4): 1149-61.

24. Neutelings G, Fénart S, Lucau-Danila A, Hawkins S. Identification and characterization of mirnas and their potential targets in flax. J Plant Physiol. 2012;169(17):1754-66.

25. Younas M, Barozai K. In silico identification of micrornas and their targets in fiber and oil producing plant flax (Linum usitatissimum L.). Pakistan J Bot. 2012;44(4):1357-62.

26. Meyers BC, Axtell MJ, Bartel B, Bartel DP, Baulcombe D, Bowman JL, Cao X, Carrington JC, Chen X, Green PJ, Griffiths-Jones S, Jacobsen SE, Mallory AC, Martienssen RA, Poethig RS, Oi Y, Vaucheret $H$, Voinnet $O$, Watanabe $Y$, Weigel D, Zhu JK. Criteria for annotation of plant MicroRNAs. Plant Cell. 2008:20(12):3186-90.

27. Ashburner M, Ball CA, Blake JA, Botstein D, Butler H, Cherry JM, Davis AP, Dolinski K, Dwight SS, Eppig JT, Harris MA, Hill DP, Tarver LI, Kasarskis A, Lewis S, Matese JC, Richardson JE, Ringwald M, Rubin GM, Sherlock G. Gene ontology: tool for the unification of biology. Nat Genet. 2000;25(1):25-9.

28. Li HY, Dong YY, Yin HL, Wang N, Yang J, Liu XM, Wang YF, Wu JY, Li XK. Characterization of the stress associated microRNAs in Glycine max by deep sequencing. BMC Plant Biol. 2011;11:170

29. Si J, Zhou T, Bo W, Xu F, Wu R. Genome-wide analysis of salt-responsive and novel microRNAs in Populus euphratica by deep sequencing. BMC Genet. 2014;15 Suppl 1:S6.

30. Tian YH, Tian YM, Luo XJ, Zhou T, Huang ZP, Liu Y, Qiu YH, Hou B, Sun D, Deng HY, Qian S, Yao KT. Identification and characterization of microRNAs related to salt stress in Broccoli, using high-throughput sequencing and bioinformatics analysis. BMC Plant Biol. 2014;14:226.

31. Bartel DP. MicroRNAs: genomics, biogenesis, mechanism, and function. Cell. 2004;116(2):281-97.

32. Han J, Fang J, Wang $C$, Yin $Y$, Sun $X$, Leng $X$, Song $C$ Grapevine microRNAs responsive to exogenous gibberellin. BMC Genomics. 2014;15:111-28.

33. Dong ZH, Shi L, Wang YW, Chen L, Cai ZM, Wang YN, Jin JB, Li X. Identification and dynamic regulation of microRNAs involved in salt stress 
responses in functional soybean nodules by high-throughput sequencing. Int J Mol Sci. 2013;14:2717-38.

34. Li MY, Wang F, Xu ZS, Jiang Q, Ma J, Tan GF, Xiong AS. High throughput sequencing of two celery varieties small RNAs identifies microRNAs involved in temperature stress response. BMC Genomics. 2014;15:242-51.

35. Gu Y, Liu Y, Zhang J, Liu H, Hu Y, Du H, Li Y, Chen J, Wei B, Huang Y. Identification and characterization of microRNAs in the developing maize endosperm. Genomics. 2013;102:472-8.

36. Ramya R, Hervé V, Jerry T, Bartel DP. A diverse and evolutionarily fluid set of microRNAs in Arabidopsis thaliana. Genes Dev. 2006;20(24):3407-25.

37. Gao ZH, Shi T, Luo XY, Zhang Z, Zhuang WB, Wang LJ. High-throughput sequencing of small RNAs and analysis of differentially expressed microRNAs associated with pistil development in Japanese apricot. BMC Genomics. 2012;13:371

38. Yang JH, Liu XY, Xu BC, Zhao N, Yang XD, Zhang MF. Identification of miRNAs and their targets using high-throughput sequencing and degradome analysis in cytoplasmic male-sterile and its maintainer fertile lines of Brassica juncea. BMC Genomics. 2013;14:9.

39. Zhai J, Dong Y, Sun Y, Wang Q, Wang N, Wang F, Liu W, Li X, Chen H, Yao N, Guan L, Chen K, Cui X, Yang M, Li H. Discovery and analysis of micrornas in Leymus chinensis under saline-alkali and drought stress using highthroughput sequencing. Plos One. 2014;9(11):e105417.

40. Jovanović Ž, Stanisavljević N, Mikić A, Radović S, Maksimović V. Water deficit down-regulates miR398 and miR408 in Pea (Pisum sativum L.). Plant Physiol Bioch. 2014;83:26-31.

41. Song JB, Gao S, Sun D, Li H, Shu XX, Yang ZM. MiR394 and LCR are involved in Arabidopsis salt and drought stress responses in an abscisic acid-dependent manner. BMC Plant Biol. 2013;13(3):1-16.

42. Turner M, Nizampatnam NR, Baron M, Coppin S, Damodaran S, Adhikari S, Arunachalam SP, Yu O, Subramanian S. Ectopic expression of miR160 results in auxin hypersensitivity, cytokinin hyposensitivity, and inhibition of symbiotic nodule development in soybean. Plant Physiol. 2013;162(4):204255.

43. Liu HH, Tian XY, Wu C, Zheng C. Microarray-based analysis of stress-regulated microRNAs in Arabidopsis thaliana. RNA. 2008;14(5):836-43.

44. Ding D, Zhang LF, Wang H, Liu ZJ, Zhang ZX, Zheng YL. Differential expression of miRNAs in response to salt stress in maize roots. Ann Bot. 2009:103(1):29-38.

45. Paul S, Kundu A, Pal A. Identification and validation of conserved microRNAs along with their differential expression in roots of Vigna unguiculata grown under salt stress. Plant Cell Tiss Org. 2011;105(2):233-42.

46. Lu SH, Sun YH, Chiang VL. Stress-responsive microRNAs in Populus. Plant J, 2008:55:131-51.

47. Barrerafigueroa BE, Gao L, Wu ZG, Zhou XF, Zhu JH, Jin HL, Liu RY, Zhu JK. High throughput sequencing reveals novel and abiotic stress-regulated micrornas in the inflorescences of rice. BMC Plant Biol. 2012;12(4):132.

48. Sunkar R, Li YF, Jagadeeswaran G. Functions of microRNAs in plant stress responses. Trends Plant Sci. 2012;17(4):196-203.

49. Khraiwesh B, Zhu JK, Zhu J. Role of miRNAs and siRNAs in biotic and abiotic stress responses of plants. Biochim Biophys Acta. 2012;1819(2):137-48.

50. Du C, Liao MS, Zhang X, Zhang FC. Regulation of copper-zinc superoxide dismutase gene expression by miR398 in Karelinia caspica under salt stress. Acta Bot Boreali-Occidentalia Sin. 2014;34(4):682-8.

51. Lu YZ, Feng Z, Bian LY, Xie H, Liang JS. Mir398 regulation in rice of the responses to abiotic and biotic stresses depends on $\operatorname{csd} 1$ and $\operatorname{csd} 2$ expression. Funct Plant Biol. 2011;38(1):44-53.

52. Cai RH, Zhao Y, Wang YF, Lin YX, Peng XJ, Li Q, Chang YW, Jiang HY, Xiang Y, Cheng BJ. Overexpression of a maize wrky58 gene enhances drought and salt tolerance in transgenic rice. Plant Cell Tiss Org. 2014;119(3):565-77.

53. Li H, Gao Y, Xu H, Dai Y, Deng DQ, Chen JM. Zmwrky33, a WRKY maize transcription factor conferring enhanced salt stress tolerances in Arabidopsis. Plant Growth Regul. 2013;70(3):207-16.

54. Yan $\mathrm{H}$, Jia $\mathrm{H}$, Chen $X$, Hao $\mathrm{L}, \mathrm{An} \mathrm{H}$, Guo $X$. The cotton WRKY transcription factor GhWRKY17 functions in drought and salt stress in transgenic Nicotiana benthamiana through ABA signalling and the modulation of reactive oxygen species production. Plant Cell Physiol. 2014:55(12):2060-76.

55. Yang X, Du Q, Chen J, Wang B, Zhang D. Association mapping in Populus reveals the interaction between Pto-miR530a and its target Pto-KNAT1. Planta. 2015;242:1-19.

56. Douglas SJ, George C, Dengler RE, Lakshmi P, Daniel RC. KNAT1 and ERECTA regulate inflorescence architecture in Arabidopsis. Plant Cell. 2002;14(3):547-58

\section{Submit your next manuscript to BioMed Central and we will help you at every step:}

- We accept pre-submission inquiries

- Our selector tool helps you to find the most relevant journal

- We provide round the clock customer support

- Convenient online submission

- Thorough peer review

- Inclusion in PubMed and all major indexing services

- Maximum visibility for your research

Submit your manuscript at www.biomedcentral.com/submit
Biomed Central 